\title{
Capillary Electrophoresis as Analytical Method for the Characterisation of Natural Diterpenoid Resins in Artistic and Historic Works: A Comparison with Gas Chromatography
}

\author{
Madeleine Dell 'mour ${ }^{\mathrm{a}}$, Anna Findeisen ${ }^{\mathrm{a}}$, Isabella Kaml ${ }^{\mathrm{a}, \mathrm{b}}$, Wolfgang Baatz ${ }^{\mathrm{b}}$ and Ernst Kenndler ${ }^{*}$, \\ ${ }^{a}$ Institute for Analytical Chemistry, University of Vienna, Währingerstr. 38, Austria \\ ${ }^{b}$ Institute for Conservation and Restoration, Academy of Fine Arts, Schillerplatz 3, Vienna, Austria
}

\begin{abstract}
We have applied capillary electrophoresis to separate and detect the characteristic main components of diterpenoic resins applied as binders in museum objects, i.e. the abietane and pimarane acids and larixol acetate. Under the influence of the applied high voltage of $15000 \mathrm{~V}$ these analytes move in a narrow separation capillary with $50 \mu \mathrm{m}$ inner diameter. This open tube has $48.5 \mathrm{~cm}$ total length, with $40.0 \mathrm{~cm}$ distance to the detector. Electrophoretic migration is supported (and, in case of the electrically neutral larixol acetate, enabled) by charged additives to the running buffer, which form ionic complexes with the analytes. The separated analytes are detected within less than 15 min by their UV absorbance measured at characteristic wavelengths of 200,250 and $270 \mathrm{~nm}$. In contrast to chromatographic methods, no derivatisation is needed. As in gas chromatography, identification of the resins is carried out by the peak patterns based on the resin composition. From the comparison with established GC methodology for the analysis of resins it can be concluded that the analytical performance parameters of the capillary electrophoretic method in terms of resolution and analysis time are comparable to those from gas chromatography, whereas the detection limit is higher in general. However, sample pretreatment is simpler for capillary electrophoresis; it consists only in the dissolution of the sample in methanol.
\end{abstract}

\section{INTRODUCTION}

Natural resins are used for a wide range of applications in many areas for cosmetic, medical and pharmaceutical purposes since ancient times. In historic and artistic works they have been used as varnishes, sealants and binders $[1,2]$. The resins are obtained directly from certain trees as exudations or plant sap (one exception is shellac, which is produced from the glandular secretion of an Indian scale insect, Laccifer lacca Kerr). Plant resins are initially viscous liquids with the higher molecular components - the actual resin being dissolved in the mono- and sesquiterpenoid essential oils. Over time these oils evaporate and the resin gradually turns into an amorphous matter.

The chemical composition of natural resins varies significantly between plant species; indeed, even the climate and the given characteristic of the soil affect it but most of them are mixtures of organic acids and esters. From the chemical point of view natural resins can be differentiated into aliphatic or aromatic compounds, respectively. Aliphatic compounds are terpenes and terpenoids; these are hemiterpenes (with a $\mathrm{C}_{5}$ skeleton), monoterpenes $\left(\mathrm{C}_{10}\right)$, sesquiterpenes $\left(\mathrm{C}_{15}\right)$, diterpenes $\left(\mathrm{C}_{20}\right)$, sesterterpenes $\left(\mathrm{C}_{25}\right)$, triterpenes $\left(\mathrm{C}_{30}\right)$, tetraterpenes $\left(\mathrm{C}_{40}\right)$, and polyterpenes. Aromatic compounds are lignanes and stilbenes.

The diterpenoids can be classified into two main groups: labdanes on the one hand and abietanes and pimaranes, on the other hand $[2,3]$. Labdanes are bicyclic molecules and

*Address correspondence to this author at the Institute for Analytical Chemistry, University of Vienna, Währingerstr. 38, Vienna, Austria;

E-mail: ernst.kenndler@univie.ac.at their compounds have a double bond containing side-chain. A typical example is larixol (or larixol acetate, see Table 1).

The abietanes are tricyclic compounds which occur in small amounts as neutral alcohols and aldehydes, but mainly as carboxylic acids (abietic acid, neoabietic acid, palustric acid and levopimaric acid). These four abietadiene acids (only varying in the positions of the double bonds) interconvert into each other when heated (e.g. for distillation) resulting in an equilibrium mixture that contents mostly abietic acid and least leavopimaric acid. The abietane with conjugated diene elements easily undergo oxidation reactions $[4,5]$. Dehydroabietic acid appears naturally in resins on the one hand, and is formed by oxidation of the four mentioned abietadiene acids on the other hand. The pimaradiene compounds (mainly as acids as well) are pimaric, isopimaric, and sandaracopimaric acid; contrary to the abietadiene compounds they possess non-conjugated double bonds.

Diterpenoid natural resins and polymers derived from them are yielded from two large families of plants, the Coniferae and the Leguminosae (also known as Fabaceae). Leguminous resins from the Leguminosae family contain either labdane type compounds or labdadiene - type acids or alcohols such as ozoic acid or zanzibaric acid. The latter are known as copals, hard resins.

Diterpenoids in conifer resins are characterized by the three main skeletal types: abietane, pimarane, and labdane (see above). These are the compounds the present paper focuses on. Abietane and pimarane type diterpenoic acids, for example abietic, dehydroabietic, pimaric and isopimaric acids, occur in substantial quantities in the resin of Pinacea. The cedar family (Cupressaceae) contains resin with mainly 
Table 1. Structural Formulae of the Analytes

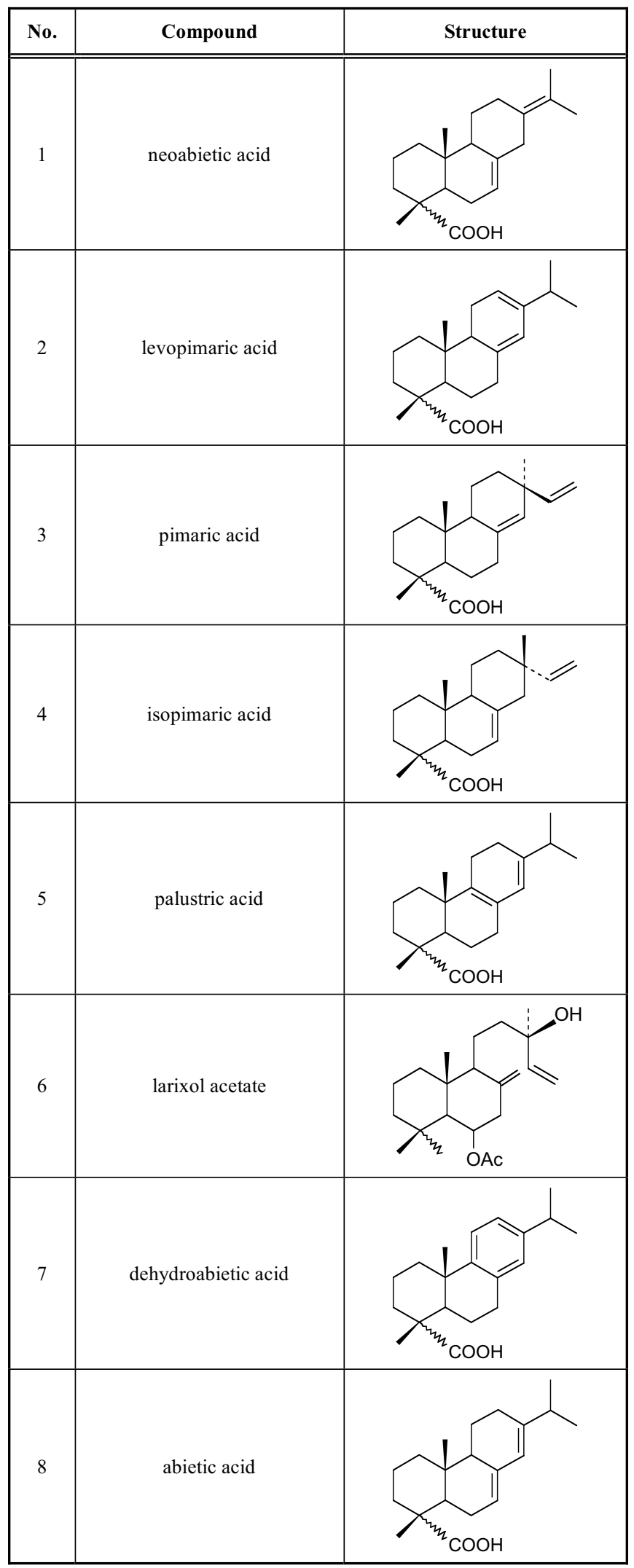

labdane-skeletal type compounds. In the resin of the araucarian family (Araucariaceae) all three skeletal types occur in similar quantities $[1,6]$.
For the analysis of resins in museum objects chromatographic methods were mostly applied, initially TLC [7]. As for gas chromatography (GC) the analytes must be volatile, the polar resin acids are normally derivatized and converted into esters before analysis. GC separation has been combined with spectrometric detection methods like Fourier transform (FT) IR spectrometry [8] or mass spectrometry (MS) [8-16]. In pyrolysis GC-MS the native resin samples are thermally decomposed in the inlet of the chromatograph, and the resulting components are separated and detected then $[12,17]$. In direct temperature MS [15-18], pyrolysis MS [19,20] and MALDI-TOF MS [21] the according substances are introduced into the MS without preceding separation with GC. Other applied methods were thermo microscopy and FTIR spectrometry [14,22], FT Raman spectrometry [23] and micro-Raman spectrometry [24].

We have successfully introduced capillary electrophoresis (CE) as an analytical method to characterise natural organic binders, including plant gums $[25,26]$, animal glues [26-28] and drying oils [29, 30] in objects of historic or artistic interest (see e.g. our recent review [31]). CE of the monomeric constituents, obtained after hydrolysis of the native samples in the usual way, was directly carried out without conversion of the analytes into more volatile or better detectable compounds, normally a pre-requisite for chromatographic separation. However, the term capillary electrophoresis summarises several methods. Briefly, in capillary zone electrophoresis (CZE) - the analogue to (elution) chromatography - the separation system is filled with a uniform buffering background electrolyte (BGE), which determines the $\mathrm{pH}$ and the ionic strength. The charged analytes move under the influence of the applied electric field, which has the same intensity in all segments of the capillary.

In the present work (which is based on two diploma thesis $[32,33])$ we use CZE - recently introduced by us in the area of binding media analysis for museum objects - as an analytical method to identify the resins which have been commonly applied in objects of the cultural heritage and compare CE with established GC methodology. As in a previous paper [34], which was, however, directed solely to CZE without considering GC analysis, we concentrate our investigation to diterpenoic resins, not to triterpenoic ones like mastic; the reason is the limited applicability of aqueous separation buffers due to the insolubility of the latter compounds. It is the goal of this paper to demonstrate that $\mathrm{CE}$ can serve as an alternative to chromatographic methods for the identification of the natural resins based on the pattern of their diterpenoic resin acids. One important constituent of at least one resin - larch turpentine - is a neutral compound and does not move electrophoretically. For this reason charged additives are used. They are intended not only to increase the solubility of the analytes and to improve the separation selectivity of the resin acid anions as introduced by Luong et al. [35], but also to serve as a vehicle to transport the neutral larixol acetate electrophoretically.

\section{MATERIALS AND METHODOLOGY}

\section{Materials}

Larixol acetate was purchased from Sequoia Research (Pangbourne, UK), the resin acids from Helix Biotech (Richmond, British Columbia, Canada). Boric acid and so- 
dium hydroxide for the background electrolyte (BGE) and methanol were from E. Merck (Darmstadt, Germany; all analytical grade). Sulfobutylether- $\beta$-cyclodextrin (Advasept 7 , SBCD) was from CyDex (Lenexa, KS, USA), methyl- $\beta$ cyclodextrin (heptakis(2,6-di-O-methyl)- $\beta$-cyclodextrin, MECD, purum) from Fluka (Steinheim, Germany). Reference material was from the collection of binding media at the Academy of Fine Arts, Vienna, Austria. The BGE was prepared with bidistilled water. Stock solutions of the standards $\left(10 \mathrm{mmol} \mathrm{L}^{-1}\right)$ were prepared in methanol, and diluted to 1 mmol L ${ }^{-1}$ with BGE prior to injection. Solvents were taken from following companies: toluene (p.a.) and methanol (HPLC-gradient grade) from VWR International (Leuven, Belgium), N,N-dimethylformamide (p.a.) from Fluka (Buchs, Switzerland). MethPrepII, ((trifluoromethylphenyl)trimethylammonium hydroxide), TFTMAH, $0.2 \mathrm{~mol}$ $\mathrm{L}^{-1}$ methanolic solution), was obtained from Alltech (Deerfield, IL, USA).

\section{Apparatus}

Electrophoretic measurements were carried out with an instrument $\left(\mathrm{HP}^{3 \mathrm{D}}\right.$, Agilent Technologies, Waldbronn, Germany) equipped with a diode array detector (DAD). UVabsorbance was measured at wavelengths between 190 and $600 \mathrm{~nm}, 10 \mathrm{~nm}$ width. Separations were carried out using uncoated fused-silica capillaries (total length $48.5 \mathrm{~cm}$, length to detector $40.0 \mathrm{~cm}$, ID $50 \mu \mathrm{m}$ I.D.; Microsolv, Long Branch, $\mathrm{NJ}$, USA). Injection was at 100 mbar.s (3.2 $\mathrm{nL}$ injection volume), separation voltage was $15 \mathrm{kV}$. The temperature of the capillary cassettes was set to $20^{\circ} \mathrm{C}$.

The BGE consisted from boric acid buffer adjusted to $\mathrm{pH}$ 9.25 (ionic strength $20 \mathrm{mmol} \mathrm{L}^{-1}$ ) with $\mathrm{NaOH}$. Solid SBCD and MECD were dissolved in the BGE at 30 and $20 \mathrm{mmol} \mathrm{L}^{-}$ ${ }^{1}$, respectively. The solutions were sonicated and filtered through $0.22 \mu \mathrm{m}$ centrifuge tube filters. Before use, new capillaries were conditioned with $1 \mathrm{~mol} \mathrm{~L}^{-1} \mathrm{NaOH}$ for about 30 $\mathrm{min}$, then with water and the running buffer, respectively, for another $30 \mathrm{~min}$ each. Before daily operation, the capillary was rinsed with $0.1 \mathrm{~mol} \mathrm{~L}^{-1} \mathrm{NaOH}$ for about $10 \mathrm{~min}$, and then with water and the BGE for 10 min each. The capillary was further conditioned by applying $15 \mathrm{kV}$ voltage for approximately $10 \mathrm{~min}$ before the first injection.

GC-MS was performed with a Hewlett-Packard 5890 gas chromatograph interfaced by an open coupling to a HewlettPackard mass-selective detector (5972 series). The chromatograph was equipped with a fused silica capillary column (30 $\mathrm{m}$ in length, $0.25 \mathrm{~mm}$ i.d.) coated with 5-HP MS (5\% phenyl, 95\% methylpolysiloxane, $0.1 \mu \mathrm{m}$ film thickness). The carrier gas was helium (purity $99.999 \%$, Air Liquid, Schwechat, Austria) at a flow rate of $0.8 \mathrm{~mL} \mathrm{~min}{ }^{-1}$. The following temperature program was used: after 1 minute at $100^{\circ} \mathrm{C}$, a first gradient of $15^{\circ} \mathrm{C} \min ^{-1}$ up to $210^{\circ} \mathrm{C}$ was applied, followed by a gradient of $2^{\circ} \mathrm{C} \mathrm{min}^{-1}$ up to $230^{\circ} \mathrm{C}$, then by a gradient of $15^{\circ} \mathrm{C} \mathrm{min}^{-1}$ up to $280^{\circ} \mathrm{C}$ (end temperature was held for 20 minutes). Injector and transfer-line temperature was $280^{\circ} \mathrm{C}$. A splitless mode was applied for the injection (injection volume was $1 \mu \mathrm{L}$ ).

Total ion monitoring scan mode was selected to record the gas chromatograms (solvent delay $4 \mathrm{~min}$ ). The operating conditions for the electron-impact mass spectrometer were: ionization voltage $70 \mathrm{eV}$, scan range from $\mathrm{m} / \mathrm{z} 35$ to 650 , scan rate 1.25 scans s$^{-1}$ and threshold 150 .

Identification was based either on the comparison with mass spectra of standards of diterpenoic acids (as methyl esters) or on the comparison with listed characteristic ions in literature applying the Mass Spectral Data Base of the Amber Search Lab, Vassar College, NY. In some cases the NIST05 database was consulted for reassurement of the search results.

\section{Sample Preparation}

Capillary electrophoresis: For dissolution of the analytes, the samples were treated with methanol at $55^{\circ} \mathrm{C}$ in an ultrasonic bath for $30 \mathrm{~min}$, then centrifuged and filtered through $0.22 \mu \mathrm{m}$ Corning ${ }^{\circledR}$ Spin-X ${ }^{\circledR}$ centrifuge tube filters (Sigma). The solution was finally diluted to the appropriate concentration with the $\mathrm{BGE}$ and directly injected into the $\mathrm{CE}$ instrument.

Gas chromatography - Mass spectrometry: about $1 \mathrm{mg}$ of sample was weighed into a $1.5 \mathrm{~mL}$ glass GC vial and 75 $\mu \mathrm{L}$ toluene together with $25 \mu \mathrm{L}$ DMF were added. The vials were sealed with aluminum-Teflon crimp caps and ultrasonicated in a water bath at $60^{\circ} \mathrm{C}$ for $30 \mathrm{~min}$. The extract was transferred into a $0.5 \mathrm{ml}$ Eppendorf vial and centrifuged for 1 min at $6000 \mathrm{rpm}$. The supernatant was pipetted into a new glass GC vial. After evaporating the solvent at a Rotavapor, the residue was derivatized with TFTMAH as described in ref. [16] by adding $20 \mu \mathrm{L}$ MethPrepII, $14 \mu \mathrm{L} \mathrm{MeOH}$ and 28 $\mu \mathrm{L}$ toluene, sealing the vials properly and leaving them in the oven for $60 \mathrm{~min}$ at $60^{\circ} \mathrm{C}$. After cooling to room temperature, the slightly yellow solutions were transferred into conical $300 \mu \mathrm{l}$ glass inserts and $1 \mu \mathrm{L}$ sample solution was injected (in the splitless mode) into the GC without dilution.

\section{RESULTS AND DISCUSSION}

Analysis by CE requires several specific properties of the sample compounds: (i) they must be sufficiently soluble in the separation medium, which is an aqueous, an organic or a mixed solvent; (ii) they must be detectable by appropriate detectors; (iii) they must migrate under the influence of the applied electric field; this means that they must be either permanent ions (from strong electrolytes), ionogenic (from weak electrolytes), or transformable into charged complexes or aggregates. For electrically neutral compounds there are several means in order to circumvent their lack in charge in practice. One possibility is the application of a charged pseudo-phase in hybrid methods like micellar electrokinetic chromatography (MEKC) or microemulsion electrokinetic chromatography (MEEKC). Another possibility is using soluble polymers added to the background electrolyte (BGE) the analytes are interacting with. These systems follow a similar separation principle like electrokinetic chromatography, and interaction with small additives can be considered formally in the same manner. Such relatively small additives are cyclodextrins, compounds commonly applied in CE because of their powerful chiral separation capability. However, they are interesting also for improved non-chiral separation, because they can form complexes with lipophilic analytes. Moreover, the according complexes of neutral analytes with charged cyclodextrins move in the electrical field and can thus be separated by CE. This strategy has been applied 
in the present work to electrophorese one main diterpenoic compound in resins - larixol acetate - together with the anionic analytes (the resin acids are dissociated at the $\mathrm{pH}$ of the BGE, as their $\mathrm{pK}_{\mathrm{a}}$ values are around 6). Most importantly, the cyclodextrines enhance the solubilisation of the sparingly soluble analytes in the separation system.

\section{Separation of Standard Compounds by $\mathrm{CE}$}

The BGE for the separation of the standard compounds consisted of borate buffer, $\mathrm{pH} 9.25$, with $20 \mathrm{mM}$ methyl- $\beta$ cyclodextrin (MECD), and $30 \mathrm{mM}$ sulfobutylether- $\beta$ cyclodextrin (SBCD) as buffer modifiers. SBCD serves as a tetraanion. This BGE has been introduced by Luong et al. [35] for the separation of resin acids including chlorinated derivatives in waste water from pulp mills. In the present work it is applied to separate the analytes of interest in natural resins consisting of abietic acid (for numbering see Table 1), dehydroabietic acid, iso-pimaric acid, levopimaric acid, neoabietic acid, palustric acid, pimaric acid, and larixol acetate.

It can be seen from the electropherogram shown in Fig. (1) that indeed all compounds are separated within $15 \mathrm{~min}$. It should be pointed out that the mutual peak height or peak area in the present electropherogram is not directly an indication of the amount of the analyte in the sample, because the response factors of the individual compounds vary strongly in case of the UV absorbance detector applied. This is caused by the different optical properties of the analytes, which possess only weakly absorbing functional groups, mainly C-C and $\mathrm{C}-\mathrm{O}$ double bonds (an exception is dehydroabietic acid, which has an aromatic ring in the molecule).

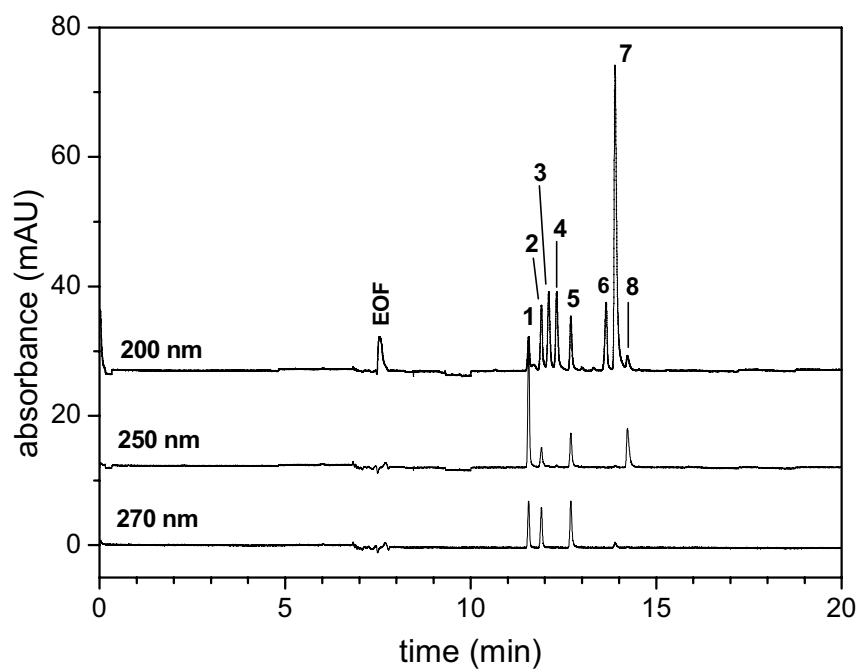

Fig. (1). Electropherogram of a standard mixture of free diterpenoic resin acids and larixol acetate. Conditions: fused-silica capillary (total length $48.5 \mathrm{~cm}$, effective length to detector $40.0 \mathrm{~cm}$, ID 50 $\mu \mathrm{m})$. Record of the UV-vis detector (diode array) at 200, 250 and $270 \mathrm{~nm}$ wavelength. Injection at 100 mbar.s, separation at $15 \mathrm{kV}$ voltage. Capillary cassette thermostatted at $20^{\circ} \mathrm{C}$. The sample (analyte concentration $1 \mathrm{mmol} \mathrm{L}^{-1}$ each) was dissolved in methanol. Separation was carried out in a BGE consisting of boric acid adjusted to $\mathrm{pH} 9.25$ (ionic strength $20 \mathrm{mmol} \mathrm{L}^{-1}$ ) with $\mathrm{NaOH}$. Concentrations of SBCD and MECD added to the BGE: $30 \mathrm{mmol} \mathrm{L}^{-1}$ and $20 \mathrm{mmol} \mathrm{L}^{-1}$, respectively. Symbols of the analytes see Table $\mathbf{1}$.
The first peak in the electropherogram of Fig. (1) is from dimethylsulfoxide, which is added to all samples as internal marker of the electroosmotic flow (EOF). The first diterpenoid compound is neoabietic acid, the last eluting one is abietic acid. Note that all analytes migrate towards the cathode, driven by the strong EOF, although their complexes with sulphated cyclodextrine are negatively charged. Baseline separation of all analytes is achieved, including that of the initially neutral larixol acetate (peak 6).

The different spectral properties of the analytes can be used as a means to confirm peak identification. For this purpose, the UV-vis spectra of the peaks are measured on-line by the use of a diode array detector. The electropherograms are then depicted for three different wavelengths, namely at 200, 250 and $270 \mathrm{~nm}$. These records are helpful to identify the compounds due to their different detection response at the different wavelengths. Larixol acetate is detectable only at $200 \mathrm{~nm}$; abietic acid does not respond at $270 \mathrm{~nm}$, and exhibits a nearly four times higher peak at $250 \mathrm{~nm}$ than at 200 $\mathrm{nm}$; the peak of neoabietic acid is also detectable at $270 \mathrm{~nm}$, and is five times higher at $250 \mathrm{~nm}$ than at $200 \mathrm{~nm}$. In contrast, palustric acid gives peaks with equal height at all three recorded wavelengths. Dehydroabietic acid has the highest response of all analytes at $200 \mathrm{~nm}$, but does not deliver a peak at 250 and $270 \mathrm{~nm}$; it can thus easily be differentiated from the abietic acid peak which is closely eluting. In contrast to pimaric acid and iso-pimaric acid, levopimaric acid responds better at 200 and $270 \mathrm{~nm}$ than at $250 \mathrm{~nm}$.

\section{CE and GC of Natural Resins}

The system used for separation of the standard diterpenoids was applied to the analysis of natural conifer resins. We have investigated different samples from colophony, Venetian, larch and Strasbourg turpentine, Burgundy pitch, Canada balsam, Congo and Manila copal, and Copaiba balsam, but show only a few examples here. In all cases, the samples were dissolved in hot methanol, centrifuged, diluted with BGE and directly injected. The electropherograms of a colophony sample is shown in Fig. (2, top panel). The main peak at $200 \mathrm{~nm}$ is from dehydroabietic acid, at $250 \mathrm{~nm}$ abietic acid gives the largest signal. Other peaks at shorter migration time can be related to palustric acid, isopimaric acid, pimaric acid and neoabietic acid, but no peak is found at the position of larixol acetate. Identification of the peaks has been carried out - in addition to the mutual signals at different wavelengths as discussed above - by standard addition.

This finding is in agreement with the composition of colophony described in the literature. We have mentioned above that there is no direct quantitation made from the relative peak areas of the analytes due to their differing optical properties. However, a detailed quantitation of the individual diterpenoids is secondary, because it is primarily the qualitative pattern which is relevant for the characterisation of the resins. The composition varies depending on a number of factors, e.g. the source, the storage conditions, or the age of the sample. It is thus more relevant for identification purposes e.g. whether in a sample larixol acetate is found or not, than what is the exact ratio e.g. of abietic acid to dehydroabietic acid. Indeed in Fig. (3, top panel) the electropherogram obtained from commercial Venetian turpentine shows the presence of larixol acetate, indicative for this type of sample. 
However, significant amounts of abietic acid and dehydroabietic acid are also recorded in this sample, which is not in agreement with literature sources. This contradiction is clarified considering the specification of this commercial Venice turpentine given by the supplier: the product is a mixture of larch turpentine with colophony. The same pattern of the resin acids (as methyl esters) are found in these samples by GC (see Figs. (2) and (3), bottom panels).
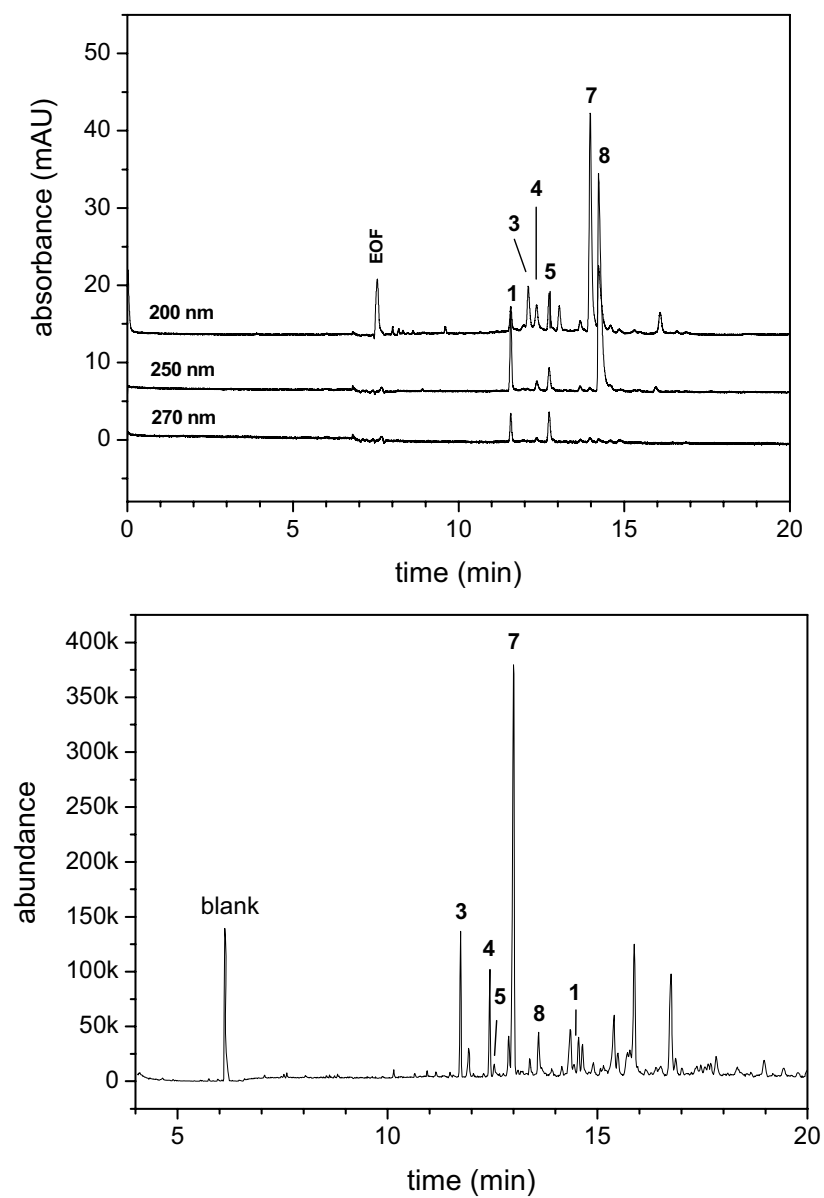

Fig. (2). Electropherogram (top panel) and gas chromatogram (bottom panel) of colophony. Experimental conditions see text. Note that in $\mathrm{CE}$ the free acids are recorded, whereas in GC the acids were derivatized to the methyl esters before analysis. Symbols of the analytes see Table 1. The peak "EOF" is a neutral marker, the peak "blank" originates from the reagents.

It is obvious that the elution order differs for the two methods, because in case of CE it is based on the mobility of the cyclodextrine complexes, in case of GC on the interaction of the methyl esters with the methylphenylsiloxane phase. It is of note that some additional peaks at higher retention time are also detected by GC, which can be identified as oxidation products by their mass spectra, a clear advantage of this method compared to CE.

We have investigated the potential of the present $\mathrm{CE}$ method for characterising the diterpenoid resins also in mixtures with other binders. Certainly the use of resinous material together with drying oils is most relevant. The result of the analysis of a 1:1 mixture of colophony and linseed oil after the sample procedure detailed in the Experimental Sec- tion (extraction with methanol, sonication, centrifugation, direct injection) shows that exactly the same pattern as with pure colophony is obtained (data not shown here), demonstrating that there is no interference from the oil constituents. It should be mentioned that in the sample pre-treatment procedure there is no hydrolysis step. This means that free fatty acids are not occurring in the samples originating from the oils.

\section{Analysis of a Modelling Mass for Reliefs on Gilded Frames from the $19^{\text {th }}$ Century}

In the $19^{\text {th }}$ century a tendency arose to substitute carved ornaments on frames by the much cheaper reliefs modelled from pasty materials, which were formed upon mixing resins, drying oils and animal glues, and adding water to obtain a paste of the appropriate constitution. This mass was pressed into forms, dried, and then released. In order to apply the ornaments on the frame, they were partly softened by the aid of hot water vapour and then applied onto the frame surface, which was gilded further. We investigated a number of samples from such frames and analysed their composition by $\mathrm{CE}$ and by GC coupled to MS for comparison.
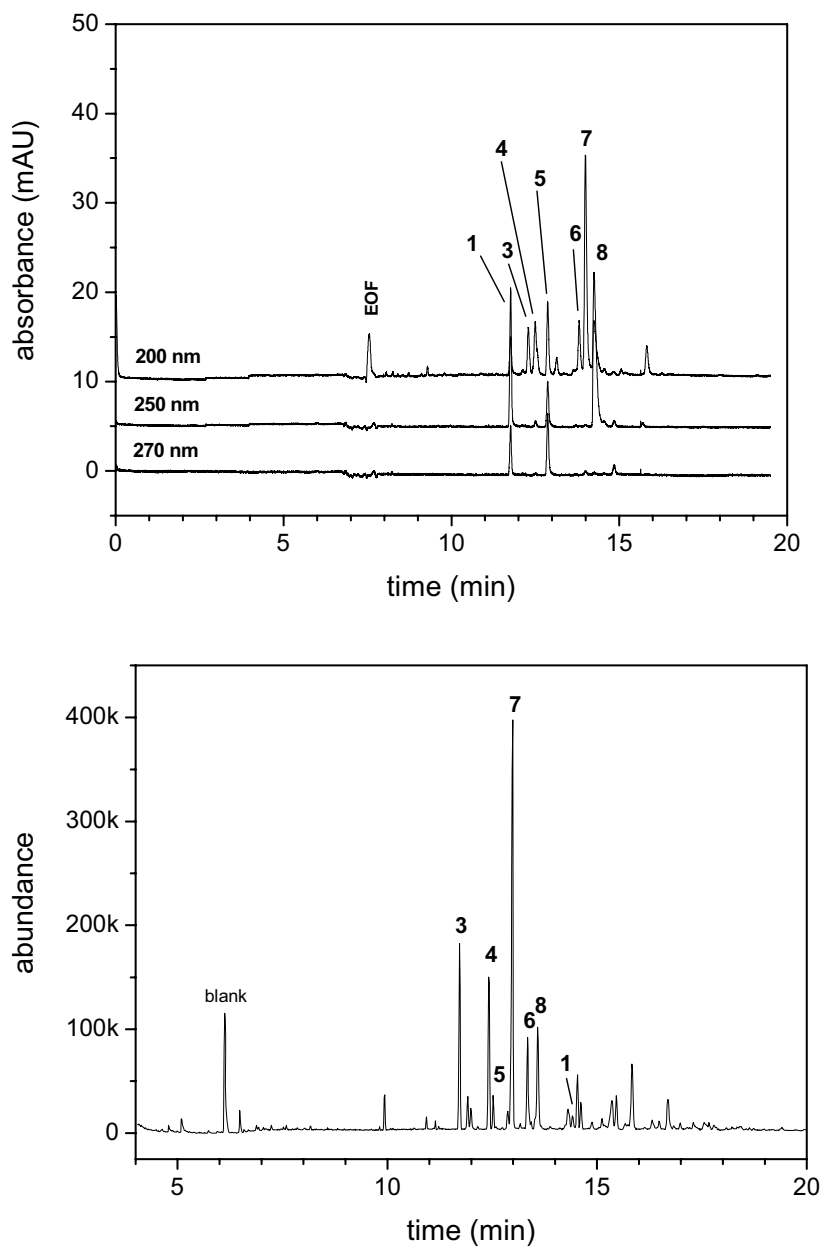

Fig. (3). Electropherogram (top panel) and gas chromatogram (bottom panel) of commercial Venetian turpentine. Conditions as in Fig. (2).

The result of the $\mathrm{CE}$ analysis obtained from one sample is shown in Fig. (4, top panel). The pattern with the main peak for dehydroabietic acid at $200 \mathrm{~nm}$ detection wave- 
length, and for abietic acid and neoabietic acid at $250 \mathrm{~nm}$ is typical for colophony. This conclusion is supported by the occurrence of palustric acid, isopimaric acid and pimaric acid. Indeed this pattern closely resembles that of colophony shown in Fig. (2). A peak within the migration time range of larixol acetate does not originate from this compound, because is gives - in contrast- also signals at 250 and $270 \mathrm{~nm}$.
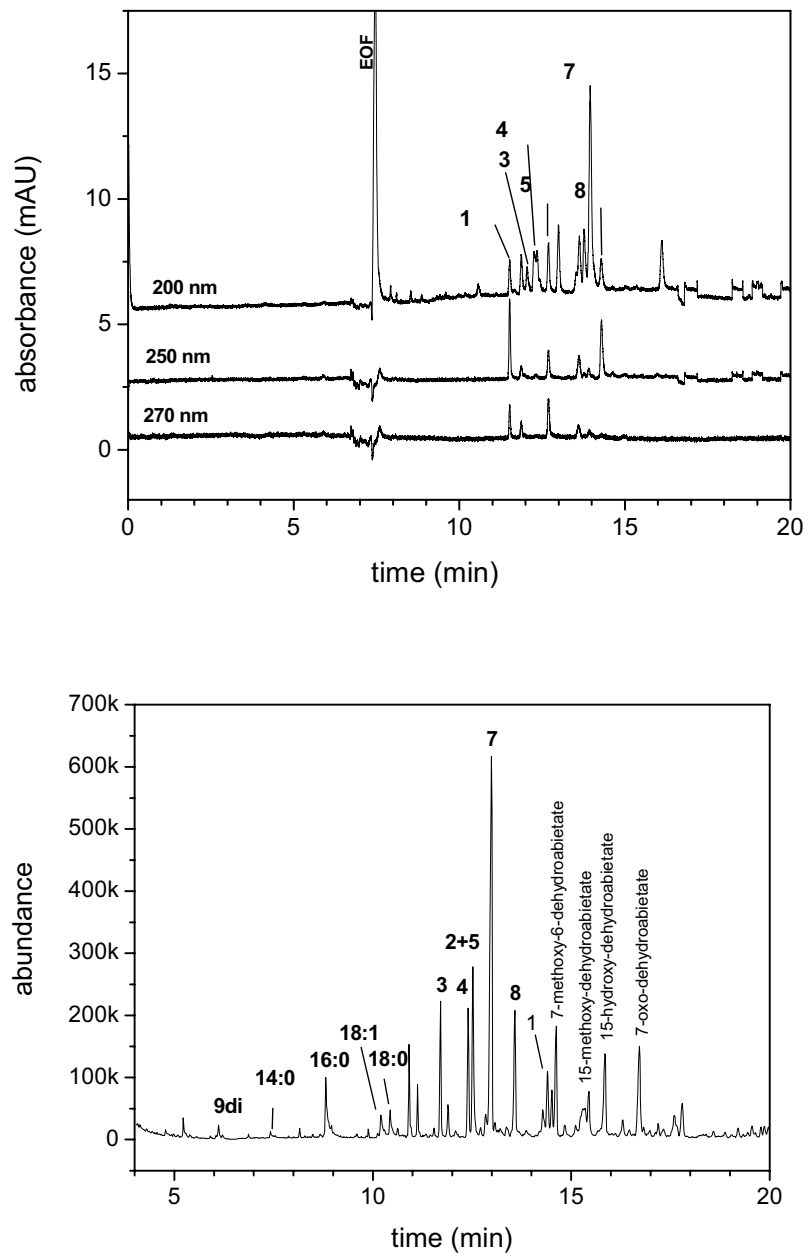

Fig. (4). Comparison of CE (top panel) with GC (bottom panel) of a sample of a gilded frame from the $19^{\text {th }}$ century. CE conditions as in Figs. (1) and (2), for GC conditions see Experimental. Peak indication see text.

The gas chromatogram obtained from the same sample after derivatisation (transesterification into the methyl esters) is shown in Fig. (4, bottom panel). It supports the findings derived from the $\mathrm{CE}$ analysis, as the same resin acids are observed.

However, GC-MS enables to identify also oxidized resin acids, for which no pure reference samples are available, by their mass spectra from the comparison with the spectra of the data library (e.g. methyl 7- and 15-methoxy- dehydroabietate, methyl 15-hydroxy-dehydroabietate and methyl 7-oxodehydroabietate, see Fig. (4)). In addition, GC allows the simultaneous determination of fatty acids most probably originating from drying oil. Methyl myristate (14:0), methyl palmitate (16:0), methyl stearate (18:0) together with methyl oleate $(18: 1)$ can be recognized, and the occurrence of a small amount of the dimethyl azelate (9di) indicates the presence of aged oil. Higher unsaturated methyl linoleate
(18:2) and methyl linolenate (18:3) are not found in the chromatogram, but this is normal in aged samples due to the polymerization and degradation of these compounds.

When CE is compared with GC, the following conclusion can be drawn. GC analysis is normally accompanied with derivatisation. This needs more manipulations, and has the additional restriction that the sample remaining after the analysis has already been transformed into the derivatives and is thus not accessible for other means. An advantage of $\mathrm{GC}$ for the present topic is that the simultaneous determination of other groups of compounds, e.g. fatty acids, or triterpenoic compounds (e.g. mastic) present in the sample is possible. However, in some cases the specifity of a method is, in contrary, considered as a positive aspect. The paramount advantage of GC is certainly the easy on-line combination with MS. This means that peak identity can be directly confirmed by the mass spectra, and moreover, identification of compounds is possible without the need of reference compounds. Finally, even for unknown compounds their structure can be elucidated. In the present topic, quantitation is easier by GC due to very similar response factors of all compounds of interest. It is thus possible to simply sum up the peak areas and to derive the relative content of the analytes in the sample from the individual peak area.

To compare the detectability of the two methods, we can take e.g. one of the acids, say dehydroabietic acid, and calculate the limit of detection (LOD) as the concentration, which gives a peak that is three times higher than the standard deviation of the background noise. As a result, in GC an LOD of $250 \mathrm{pg} \mathrm{LL}^{-1}$ sample solution is obtained for methyl dehydroabietate. Time of analysis might be longer by GC because due to the temperature program the initial temperature must be re-established, which adds to the analysis time. This aspect is more relevant only for routine analysis with a large sample through-put.

$\mathrm{CE}$ analysis is cheaper, as only diluted aqueous solution of electrolytes and additives are needed as BGE. A low consumption of chemicals is evident (the column volume is only few $\mu \mathrm{L}$, injection volume only few $\mathrm{nL}$ ). The separation column is simply an open tube without stationary phase. The overall costs of the instrumentation are comparable with GC (without MS). Concerning laboratory work, no derivatization is needed, as direct analysis after dissolution of the analytes is possible. On the other hand, there is a restricted possibility for on-line combination with MS (this is expensive, skillful, and has severe limitations in compatibility). The sensitivity of CE depends on the detected property. Fluorescence detection is sensitive, especially when undertaken by laser induced fluorescence (LIF). The present analytes have no fluorophors, and possess low UV absorbance, so their detectability with the UV-absorbance detector (the common $\mathrm{CE}$ detector) is moderate to low. Due to the different spectral properties of the present compounds quantitation needs individual response factors, which is also not favorable. For comparison of the LOD with GC we select again dehydroabietic acid. It is obvious that this analyte will deliver the most favorable results due to its high UV absorbance at 200 $\mathrm{nm}$. For this compound the LOD is as low as $1.4 \mu \mathrm{mol} \mathrm{L} \mathrm{L}^{-1}$. Expressed in weight it is about $400 \mathrm{pg}$ per $\mu \mathrm{L}$ sample solution; it follows that for this analyte the detectability of CE is comparable to $\mathrm{GC} / \mathrm{MS}$, whereas slightly lower. Interesting is, 
however, the much better detectibility of $\mathrm{CE}$ when expressed in amount per injected volume. As this is only less than $4 \mathrm{~nL}$ in the present case (it is $1 \mu \mathrm{L}$ in GC), we can detect the minute amount of less than $2 \mathrm{pg}$.

\section{CONCLUSIONS}

Capillary electrophoresis was applied as an analytical method for the characterisation and identification of different diterpenoic resins used in museum objects. As for CE of animal glues, plant gums and drying oils, no further derivatisation was needed prior to analysis, in contrast to most chromatographic methods. Sample pre-treatment consists only in dissolution in methanol. With the separation systems used no interference in the identification of the analytes from the constituents of the other binders was observed. Analysis time is in the same range of chromatographic analysis. For the given conditions, detectability of CE by UV absorbance is comparable with $\mathrm{GC} / \mathrm{MS}$ for the compounds with highest absorbance, but lower for most analytes.

\section{ACKNOWLEDGEMENTS}

The authors thank P. Frühauf, and V. Pitthard from the Kunsthistorisches Museum, Vienna, for their kind support with GC analyses.

\section{REFERENCES}

[1] Mills, J. S.; White, R. Stud. Conserv., 1977, 22, 12.

[2] Mills, J. S.; White, R. The Organic Chemistry of Museum Objects, 2nd ed.; Butterworth Heinemann: London, 1994.

[3] Sandermann, W. Naturharze Terpentinöl Tallöl; Springer: Berlin, 1960.

[4] van den Berg, K. J.; Boon, J. J.; Pastorova, I. I.; Spetter, L. F. J. Mass Spectrom., 2000, 35, 512.

[5] Scalarone, D.; Lazzari, M.; Chiantore, O. J. Anal. Appl. Pyrol., 2002, 64, 345

[6] Langenheim, J. H. Plant resins: chemistry, evolution, ecology, and ethnobotany; Timber Press: Portland, 2003.

[7] Kharbade, B. V.; Srivastava, N.; Joshi, G. P.; Agrawal, O. P. J. Chromatogr., 1988, 439, 430 .

[8] Kenndler, E.; Mairinger, F. Fresenius J. Anal. Chem., 1990, 338, 635
[9] Colombini, M. P.; Modugno, F.; Giannarelli, S.; Fuoco, R.; Matteini, M. Microchem. J., 2000, 67, 385.

[10] Watts, S.; de la Rie, E. R. Stud. Conserv., 2002, 47, 257.

[11] Osete-Cortina, L.; Domenech-Carbo, M. T.; Mateo-Castro, R.; Gimeno-Adelantado, J. V.; Bosch-Reig, F. J. Chromatogr. A, 2004, 1024, 187.

[12] de la Cruz-Canizares, J.; Domenech-Carbo, M. T.; GimenoAdelantado, J. V.; Mateo-Castro, R.; Bosch-Reig, F. J. Chromatogr. A, 2005, 1093, 177 .

[13] Andreotti, A.; Bonaduce, I.; Colombini, M. P.; Gautier, G.; Modugno, F.; Ribechini, E. Anal. Chem., 2006, 78, 4490.

[14] Domenech-Carbo, M. T.; Kuckova, S.; de la Cruz-Canizares, J.; Osete-Cortina, L. J. Chromatogr. A, 2006, 1121, 248.

[15] Modugno, F.; Ribechini, E.; Colombini, M. P. Rapid. Commun. Mass Spectrom., 2006, 20, 1787.

[16] Pitthard, V.; Griesser, M.; Stanek, S.; Bayerova, T. Macromol. Symp., 2006, 238, 37.

[17] Chiavari, G.; Fabbri, D.; Prati, S. Chromatographia, 2002, 55, 611

[18] Scalarone, D.; van der Horst, J.; Boon, J. J.; Chiantore, O. J. Mass Spectr., 2003, 38, 607.

[19] Wright, M. M.; Wheals, B. B. J. Anal. Appl. Pyrolysis, 1987, 11, 195.

[20] Osete-Cortina, L.; Domenech-Carbo, M. T. J. Chromatogr. A 2005, 1065, 265 .

[21] Scalarone, D.; Duursma, M. C.; Boon, J. J.; Chiantore, O. J. Mass Spectrom., 2005, 40, 1527.

[22] Odlyha, M. Thermochim. Acta, 1995, 269/270, 705.

[23] Edwards, H. G. M.; Farwell, D. W.; Daffner, L. Spectrochim. Acta, Part A: Mol. Biomol. Spectrosc., 1996, 52A, 1639.

[24] Vandenabeele, P.; Wehling, B.; Moens, L.; Edwards, H.; De Reu, M.; Van Hooydonk, G. Anal. Chim. Acta, 2000, 407, 261.

[25] Größ1, M.; Harrison, S.; Kaml, I.; Kenndler, E. J. Chromatogr. A 2005, 1077, 80 .

[26] Mazanek, M.; Kaml, I.; Kenndler, E. Stud. Conserv., 2006, 51, 130.

[27] Kaml, I.; Vcelakova, K.; Kenndler, E. J. Sep. Sci., 2004, 27, 161.

[28] Harrison, S.; Kaml, I.; Prokoratova, V.; Mazanek, M.; Kenndler, E. Anal. Bioanal. Chem., 2005, 382, 1520 .

[29] Surowiec, I.; Kaml, I.; Kenndler, E. J. Chromatogr. A, 2004, 1024, 245.

[30] Harrison, S.; Kaml, I.; Rainer, F.; Kenndler, E. J. Sep. Sci., 2005 , $28,1587$.

[31] Kaml, I.; Kenndler, E. Curr. Anal. Chem., 2007, 3, 33

[32] Findeisen, A. Diploma Thesis, University Vienna, Vienna, 2007.

[33] Dell mour, M. Diploma Thesis, University Vienna, Vienna, 2007.

[34] Findeisen, A.; Kolivoška, V.; Kaml, I.; Baatsch, W.; Kenndler, E. J. Chromatogr. A, 2007, 1157, 142.

[35] Luong, J. H.; Rigby, T.; Male, K. B.; Bouvrette, P. Electrophoresis, 1999, 20, 1546. 